\title{
What is winter? Modelling spatial variation in bat host traits and hibernation and their implications for overwintering energetics
}

\author{
C. Hranac ${ }^{1}$, Catherine Haase ${ }^{2}$, Nathan Fuller ${ }^{3}$, Meredith McClure ${ }^{4}$, Jonathan Marshall ${ }^{1}$, \\ Cori Lausen $^{5}$, Liam McGuire ${ }^{6}$, Sarah Olson ${ }^{7}$, and David Hayman ${ }^{1}$ \\ ${ }^{1}$ Massey University \\ ${ }^{2}$ Montana State University System \\ ${ }^{3}$ Texas Tech University \\ ${ }^{4}$ Conservation Science Partners \\ ${ }^{5}$ Wildlife Conservation Society Canada \\ ${ }^{6}$ University of Waterloo \\ ${ }^{7}$ Wildlife Conservation Society
}

January 28, 2021

\begin{abstract}
White-nose syndrome (WNS) has decimated hibernating bat populations across eastern and central North America for over a decade. Disease severity is driven by the interaction between bat characteristics, the cold-loving fungal agent, and the hibernation environment. While we further improve hibernation energetics models, we have yet to examine how spatial heterogeneity in host traits is linked to survival in this disease system. Here we develop predictive spatial models of body mass for the little brown myotis (Myotis lucifugus) and reassess previous definitions of the duration of hibernation of this species. Using data from published literature, public databases, local experts, and our own fieldwork, we fit a series of generalized linear models with hypothesized abiotic drivers to create distribution-wide predictions of pre-hibernation body fat and hibernation duration. Our results provide improved estimations of hibernation duration and identify a scaling relationship between body mass and body fat; this relationship allows for the first continuous estimates of pre-hibernation body mass and fat across the species' distribution. We used these results to inform a hibernation energetic model to create spatially-varying fat use estimates for M. lucifugus. These results predict that WNS mortality of newly and soon-to-be infected M. lucifugus populations in western North America may be comparable to the substantial die-off observed in eastern and central populations.

What is winter? Modelling spatial variation in bat host traits and hibernation and their implications for overwintering energetics

C. Reed Hranac ${ }^{1 ¥}$, Catherine G. Haase ${ }^{2+}$, Nathan W. Fuller ${ }^{3 \$}$, Meredith L. McClure ${ }^{4}$, Jonathan C. Marshall $^{5}$, Cori L. Lausen ${ }^{6}$, Liam P. McGuire ${ }^{3^{\wedge}}$, Sarah H. Olson ${ }^{7}$, David T. S. Hayman ${ }^{1}$

${ }^{1}$ Molecular Epidemiology and Public Health Laboratory, Hopkirk Research Institute, Massey University, Palmerston North, New Zealand

2 Department of Microbiology and Immunology, Montana State University, Bozeman, USA.

${ }^{3}$ Department of Biological Sciences, Texas Tech University, Lubbock, USA

${ }^{4}$ Conservation Science Partners, Truckee, USA

${ }^{5}$ Institute of Fundamental Sciences, Massey University, Palmerston North, New Zealand
\end{abstract}


${ }^{6}$ Wildlife Conservation Society Canada, Toronto, Canada

7 Wildlife Conservation Society, Health Program, Bronx, USA

*Corresponding author:crh244@gmail.com

${ }^{¥}$ Current affiliation: Colorado Department of Public Health and Environment, Healthcare Associated Infections and Antimicrobial Resistance Program, Denver, USA

${ }^{+}$Current affiliation: Department of Biology, Austin Peay State University, Clarksville, USA

${ }^{\$}$ Current affiliation: Texas Parks and Wildlife Department, Nongame and Rare Species Program, Austin, USA

Waterloo, Ontario, Canada

\begin{abstract}
White-nose syndrome (WNS) has decimated hibernating bat populations across eastern and central North America for over a decade. Disease severity is driven by the interaction between bat characteristics, the cold-loving fungal agent, and the hibernation environment. While we further improve hibernation energetics models, we have yet to examine how spatial heterogeneity in host traits is linked to survival in this disease system. Here we develop predictive spatial models of body mass for the little brown myotis (Myotis lucifugus ) and reassess previous definitions of the duration of hibernation of this species. Using data from published literature, public databases, local experts, and our own fieldwork, we fit a series of generalized linear models with hypothesized abiotic drivers to create distribution-wide predictions of pre-hibernation body fat and hibernation duration. Our results provide improved estimations of hibernation duration and identify a scaling relationship between body mass and body fat; this relationship allows for the first continuous estimates of prehibernation body mass and fat across the species' distribution. We used these results to inform a hibernation energetic model to create spatially-varying fat use estimates for $M$. lucifugus. These results predict that WNS mortality of newly and soon-to-be infected M. lucifuguspopulations in western North America may be comparable to the substantial die-off observed in eastern and central populations.
\end{abstract}

\title{
Keywords
}

Hibernation energetics, Myotis lucifugus, Pseudogymnoascus destructans, white-nose syndrome, winter duration

\section{INTRODUCTION}

In temperate zones of North America, hibernating animals, includingMyotis lucifugus (little brown myotis), bridge resource poor winters through energetic budgeting and behavioural changes (Hock 1951, Wang 1978, Speakman and Rowland 1999, Ruf and Geiser 2015). Survival over winter hibernation depends upon three main facets: 1) the amount of energy stored, primarily in body fat, 2) energetic expenditure (rate of metabolic consumption), and 3) the duration of hibernation (Humphries et al. 2002). Hibernation is composed of bouts of torpor, during which body temperature drops to near ambient temperature to limit heat loss and results in reduced metabolism to restrict the consumption of finite metabolic resources. Torpor is periodically interrupted by energy intensive periods of arousal during which hibernators return to euthermic body temperature (Hayman et al. 2010). Hibernators arouse for a variety of proposed reasons (for a review see Carey [2019] and citations within), including the need to eliminate metabolic waste, regain water balance, or mate. While arousals represent a small fraction of the total time spent in hibernation, they account for the majority of energy consumed, with a single arousal costing as much as $5 \%$ of total overwinter energetic costs (Thomas et al. 1990).

Microclimate selection is critical for hibernators (Boyles et al. 2007). By allowing body temperature to drop during torpor, $M$. lucifugusconsumes roughly 80 -fold less energy per unit time due to the relationship between metabolic rate and temperature (Hock 1951, Speakman and Thomas 2003). To maximize the utility of these metabolic reductions, bats seek out caves, mines, scree slopes, or other locations generally referred to 
as "hibernacula" where they can overwinter (Speakman and Thomas 2003). Relative to the surface landscape, subterranean locations can provide suitable low temperature (i.e., $0-10 @ \mathrm{C}$ ) habitats for hibernators (Thomas and Cloutier 1992). Microclimate selection will vary greatly based on species-specific preferences (Haase et al. 2021), and roost selection within the larger hibernaculum critically affects both the frequency of arousals and efficiency of torpor (Humphries et al. 2002, Czenze et al. 2013, Haase et al. 2019). Species such as $M$. lucifugus appear to choose roost locations with stable, nearly saturated environments and low temperatures to ameliorate their relatively high rates of evaporative water loss, while other bat species are capable of using more arid, and less thermally-stable, roosts as hibernacula (Klüg-Baerwald and Brigham 2017, Klüg-Baerwald et al. 2017). Even so, within a cave or mine system, roost conditions may not remain stationary throughout the duration of hibernation and some bats will relocate within the hibernaculum to seek specific microclimate conditions as their body condition changes (Hayman et al. 2010).

The duration of winter hibernation is another critical determinant to the survival of hibernators. The stimuli that drive immergence (entrance) to and emergence (exit) from hibernation, and their geographic variation, are under-described (Norquay and Willis in press, Lane et al. 2012, Czenze et al. 2013). The duration of winter hibernation presents a strong selective pressure, as longer winters result in shorter growing seasons and less time available for pre-hibernation fattening (Kunz et al. 1998). While animals that hibernate at more southern latitudes may be able to capitalize on breaks in the winter weather to opportunistically feed (Thomas et al. 1990), this is not always an option for other latitudes. Entrances to hibernacula may be blocked by snow, preventing foraging even if breaks in the weather did allow for the re-emergence of prey species (CLL, unpublished data). Because North American hibernating bats feed on insects, researchers have estimated effective hibernation duration based upon the number of freezing days, with the assumption that freezing temperatures prevent insect availability until spring (Humphries et al. 2002, Hayman et al. 2016). However, some populations of bats may emerge from hibernation while freezing temperatures are still present, suggesting that there may be more complex determinants of emergence times (Johnson et al. 2017). Additionally, site-level differences, such as slope aspect, foliage cover, and proximity to water may influence the density of prey insects and their ability to persist on the landscape.

Beyond the normal challenges of hibernation, the epizootic white-nose syndrome (WNS), caused by the psychrophilic fungusPseudogymnoascus destructans, has increased energetic demands for hibernating bats (Blehert et al. 2009). The fungal pathogen responsible for the disease has spread rapidly through North America since 2006 and has killed millions of hibernating bats (Frick et al. 2015). Bats can be exposed to the fungus during the swarming period or over hibernation in the hibernacula. Once infected, the fungus grows during hibernation when bat skin temperatures are reduced and immune function is suppressed (Verant et al. 2012, Langwig et al. 2015, 2016). While there is still discuss the ultimate cause of mortality in WNSimpacted bats, it has been linked to the increased frequency of arousals in infected bats, ultimately resulting in the depletion of fat stores prior to the end of the hibernation period and subsequent starvation (Warnecke et al. 2012, Lilley et al. 2016).

There has been much research on energy consumption over hibernation in multiple bat species (Thomas et al. 1990, Cryan and Wolf 2003, Willis et al. 2006, Jonasson and Willis 2012, McGuire et al. 2014, Haase et al. 2019) and across the distribution of single bat species (Humphries et al. 2002, Hayman et al. 2016), but we have yet to determine how the spatial variation in the other two critical parameters governs overwinter survival for hibernators: duration of winter hibernation and amount of fat stores taken into hibernation. Previous models (Hayman et al. 2016) allowed for spatial variation in winter duration; however, the definition of winter duration was made a priori and based solely upon the number of nights with an average temperature below $0^{\circ} \mathrm{C}$ (Humphries et al. 2002). Similarly, the amount of body fat has generally been fixed as 25-30\% of total body mass in most studies (Humphries et al. 2002, Hayman et al. 2016, Haase et al. 2021). Fat resources are a major determinant of survival (Haase et al. 2019) and thus this assumption of proportion of body fat warrants review. Here we used generalized linear and linear models to: 1) estimate hibernation duration, 2) relate body mass to pre-hibernation fat stores, and 3) predict pre-hibernation body mass and fat across the distribution of M. lucifugus. We focused on M. lucifugus due to the high impact of WNS on $M$. lucifugus populations, its widespread distribution, and the availability of published data. We predicted that 
spatial variation in overwinter duration would drive variation of body mass and fat to account for different energy requirements leading to spatial variation in WNS disease outcomes. Finally, we used a mechanistic model of hibernation energetics (Haase et al. 2019) to estimate the total metabolic costs of hibernation with and without the impacts of WNS across the species' distribution of M. lucifugus .

\section{METHODS}

\section{Hibernation duration data}

We compiled hibernation duration data from literature, publicly available datasets, and our own field data. We also solicited data-based estimates of hibernation duration from local bat researchers across North America where few records were available from other sources (Table S1, Figure S1). We restricted the study region for this analysis to temperate North America (above the Tropic of Cancer and below the Arctic Circle), although the published range of M. lucifugus extends into the Arctic (Fenton and Barclay 1980). Spatially explicit records of immergence and emergence collected from the literature generally reported the average day of entrance or emergence, although some data were presented only as the duration of hibernation. Where sex-specific dates were given for a location, dates were averaged as insufficient data existed to complete a sex-specific analysis.

Acoustic bat recorders (Songmeter SM2+BAT; Wildlife Acoustics, MA, USA) were deployed by Wildlife Conservation Society Canada (WCS-C) to record bat activity across western Canada between 2008 and 2016. Microphones (either SMX-US or SMX-U1) were placed on 12-18-foot-tall telescoping poles above likely hibernation or commuting areas (e.g. riverbanks, cliff ridge tops) starting as early as mid-August. Acoustic activity was typically recorded throughout the duration of the winter and data were retrieved between midMarch and mid-May. Recordings were manually analysed using AnalookW software (Titley Scientific, Inc.) and customized noise-filters were used to pull files containing bat pulses. The occurrence of at least 2 bat echolocation pulses in a file was required to identify the recording as a 'bat pass', and the number of passes were summed nightly. The start of the hibernation period was defined by the last 3-night window between August 15 and December 31, in which [?]10 passes were identified, while the end of the hibernation period was defined by the first 3-night window between March 1 and May 15 in which [?]10 passes were recorded (Lausen and Barclay 2006). In locations where bat activity was consistently low throughout the year (spring and fall nightly activity often failing to exceed 10 passes), hibernation start and end points were defined by date of the last and first bat recordings, respectively. For sites with multiple years of data, we took the mean immergence and emergence dates.

\section{Pre-hibernation fat and body mass data}

The data required to directly assess the spatial variation in body fat for M. lucifugus prior to hibernation do not exist, so we relied upon scaling fat mass with spatially-varying body mass. We used body composition datasets obtained by quantitative magnetic resonance (QMR; McGuire and Guglielmo 2010) measurements that provided both pre-hibernation fat mass and body mass information from multiple $M$. lucifugus populations, including New York, Vermont (McGuire et al. 2018) and Montana (Haase et al. 2019). We tested for differences in fat among locations using a linear model with fat as a response variable. Given that there was no significant difference among locations, we then fit a linear model with fat as the response and body mass as the predictor variable to predict fat mass from body mass (French 1985). This fitted relationship then allowed us to predict fat mass at locations where we only had body mass measurements. We gathered additional body mass data from the literature and VertNet (vertnet.org), in which records were filtered to include only those with geographic location, body mass, and recorded between September and December (Table S2, Figure S1).

\section{Prediction of spatially-varying hibernation duration and fat mass}

Rather than photoperiod-based metrics, we used the availability of food resources to predict the onset of hibernation, including climatic variables due to the correlation between ambient temperature and insect activity (Mellanby 1939). The first (herein Original model) definition of hibernation duration (Humphries 
et al. 2002) was the number of days per year where mean nightly temperature from 12 a.m. to 6 a.m. was below freezing. The alternative spatial covariate layers we assumed to be related to the duration of winter were: degrees latitude North (Northing), elevation (DEM ), number of days of frost annually (Daysfrost), number of days with an average temperature below freezing annually (Daysfreezing), and number of days outside of the growing season annually (Days grow $)$. Climate data were generated with the ClimateNA v5.10 software package based on methodology described by Wang et al. (2016). All spatial data were re-projected to the same $1 \mathrm{~km}^{2}$ resolution, cropped, and masked to the study extent (Frick and Hijmans 2017).

All covariates were regressed against the estimated duration of hibernation in univariate models and in multivariate models adjusting for latitude (Northing) and/or elevation (DEM). Models were assessed through a two-step process: initial linear models were cross-validated using Holm's method (Holm 1979) to identify outliers and generalized linear models were then fit to the modified data set. From initial model fits, externally studentized residuals were converted to p-values and adjusted for multiple testing using Holm's method. Observations with adjusted p-values $(<0.5)$ across multiple models were excluded, and subsequent models refitted using reduced data. Generalized linear models were then fit with the modified data set and model selection was assessed by Akaike information criterion (AIC; (Burnham and Anderson 2002).

To predict hibernation duration over the study region using the best model selected by AIC, we first assessed model residuals for spatial auto-correlation using Moran's I from the spdep R package (Bivand and Wong 2018). When no autocorrelation was identified, the top model by AIC was predicted across the study extent using the spatial layers to create a continuous estimate of hibernation duration across the species' distribution. If autocorrelation was detected, a spatially-weighted generalized linear model was generated using the glmmfieldspackage (Anderson and Ward 2019) for the top model by AIC and then hibernation duration was projected across the species' distribution.

Since these proposed spatial covariates defined the annual inactive period, we also assume that they were likely to drive spatial variation in body mass as bats would need increased metabolic resources to survive longer durations of hibernation. We repeated the above model fitting and selection methods of the same independent variables against the response variable of pre-hibernation body mass across all data collection locations. We then assessed the top model for spatial autocorrelation and predicted body mass across the species' distribution. Finally, we predicted spatially-varying pre-hibernation fat mass across the species' distribution using the scaling equation fit above.

\section{Prediction of spatially-varying hibernation survival}

We used a mechanistic hibernation energetics model to estimate the total cost of hibernation (in grams of fat) for a hibernating $M$. lucifugus across its distribution (see Haase et al. (2019) for complete model documentation). The model is dynamic and species-specific, using metabolic and morphometric parameters to estimate the amount of fat used during hibernation. We used published parameter values for M. lucifugus (Haase et al. 2019) with the exception of arousal duration, which was set to $2.2 \mathrm{~h}$ (CLL, unpublished data).

These energetic functions are dependent on temperature and relative humidity of the roost to provide estimates of energy expenditure across a wide range of potential conditions. The temperature and humidity dependent growth of $P$. destructans can be included in the model to estimate the metabolic impact of infection on energy consumption as the fungal load increases. We modelled the total energetic costs of hibernation with and without the impacts of $P$. destructans across two hibernaculum microclimate scenarios. First, we used fixed microclimate conditions that assumed a bat could access the preferred optimal hibernaculum microclimate conditions across the species' distribution. We used conditions of $4 \mathrm{degC}$ and $98 \%$ relative humidity based on observations reported in the literature (Table S3). These microclimatic conditions are thought to provide for the longest possible hibernation duration, a hypothesis supported by recent findings (Haase et al. 2019). Second, we used a spatially-explicit model of subterranean temperature conditions to predict the best available (i.e., closest to optimal) temperature at a given location. This approach assumes that bats will select roosts within hibernacula that offer their preferred temperature when possible but will likely tolerate warmer or cooler temperatures when necessary, especially at the range margins. Unfortu- 
nately, the not much information exists for relative humidity; therefore, we used the optimal $98 \%$ relative humidity for both the optimal and best available temperature scenarios.

To estimate the closest available temperature to the optimal temperature at any given location, we used a spatially explicit model of subterranean winter temperatures (McClure et al. 2020). This model estimates subterranean winter temperature should a potential hibernaculum exist based on mean annual surface temperature, site type (cave or mine), distance from the site entrance, and several less influential predictors representing topography, land cover, and presence of water. The model predicts an increase in subterranean temperature with increasing mean annual surface temperature and distance from the site entrance and higher temperatures in mines than in caves. For each $1 \mathrm{~km}^{2}$ cell we estimated minimum and maximum roosting temperatures likely to be present within a hibernaculum using methods described in McClure et al. 2020. We then assigned each raster cell the best available temperature; either the preferred temperature if that temperature was predicted to be available given the minimum and maximum temperature estimations, or the closest temperature available to the preferred roost temperature.

Finally, we estimated the amount of fat required to survive hibernation for each of our $1 \mathrm{~km}^{2}$ cells for both healthy and WNS-impacted bats by using the dynamic hibernation energetics model and our spatially-varying predictions of hibernation duration and fat. We determined if an average bat could survive hibernation for each cell by subtracting the predicted fat required to survive the hibernation duration from the fat available prior to hibernation. Positive values indicate a bat's ability to survive hibernation with excess fat, while negative values indicate the depletion of fat stores prior to the end of the hibernation period. We compared the predicted amount of fat required by healthy bats against the fat required by infected bats to estimate the relative increase of energetic costs of $P$. destructans on $M$. lucifugus as a percentage (the difference between resources required to survive hibernation when infected and when healthy in grams of fat, divided by grams of fat required to survive hibernation as healthy bat, multiplied by 100). We also translated the amount of fat "leftover" after the conclusion of hibernation into days post hibernation, i.e., how long the bat could continue hibernating, for both healthy and infected bats.

All analyses were performed in R (R Development Core Team 2009), with spatial handling tools from raster (Hijmans 2016) and sppackages (Pebesma and Bivand 2005).

\section{RESULTS}

\section{Hibernation duration}

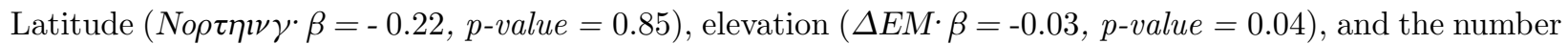
of days in frost $\left(\Delta a \psi_{S_{\varphi \rho o \sigma \tau}} \cdot \beta=0.83, p\right.$-value $\left.<0.001\right)$ best predicted hibernation duration across the study area $(A I C c=489.29$; Table S4). There was no spatial correlation of these predictions (Moran's $I=-0.0036$, $p$-value $=0.354)$ and thus no spatial corrections were needed. The selected model outperformed the original a priori estimate of the duration of winter (Original, [?]AIC 19.06).

Given the best model, median predicted hibernation duration was $\sim 179$ days $($ mean $=169.16, s d=45.36)$. Maximum hibernation duration across the study extent was estimated at $\sim 289$ days in the upper portions of Manitoba, Ontario, and Quebec, 45 days longer than the longest observation in the training data (from Manitoba, Canada, 19; Figure 2). Despite this, $95 \%$ of all cells had a predicted hibernation duration below 225 days, and only $5 \%$ were below 80 days (Table S5).

\section{Body mass}

Pre-hibernation fat stores were strongly related to body mass $\left(\beta=0.597, F_{1,171}=826.7, p=<0.001\right.$,

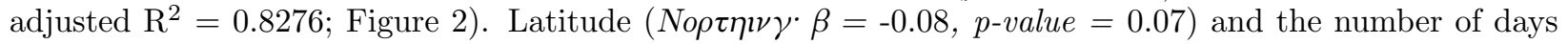
below freezing $\left(\Delta a \psi_{\varphi \rho \epsilon \epsilon \zeta \epsilon} \cdot \beta=0.04, p\right.$-value $\left.<0.001\right)$ best predicted fat mass across the species' distribution $(A I C c=162.57$; Table S4). However, the model including latitude, number of annual days below freezing, and elevation performed similarly $\left(\Delta A I^{\prime \prime}<2\right.$; Table S4).

Given the best model, the median predicted pre-hibernation body mass across the species' distribution of 
M. lucifugus was $8.65 \mathrm{~g}$ ( mean $=9.14 \mathrm{~g}, s d=1.84 \mathrm{~g}$ ) and $95 \%$ of the cells predicted values between $7.04 \mathrm{~g}$ and12.52 g. Median pre-hibernation fat stores were predicted at $2.32 \mathrm{~g}$ ( mean $=2.61 \mathrm{~g}$, sd $=1.10 \mathrm{~g})$ with $95 \%$ of cells predicting available fat available ranging between $1.36 \mathrm{~g}$ and $4.63 \mathrm{~g}$.

\section{Overwinter hibernation survival}

Overall, our energetic model predicted nearly ubiquitous survival of uninfected bats throughout the range of M. lucifugus with $<0.0001 \%$ of cells falling below the threshold for survival. Our results found that $95 \%$ of all uninfected bats roosting at $4 @ \mathrm{C}$ and $98 \%$ relative humidity during hibernation would only require 0.21 $\mathrm{g}-0.60 \mathrm{~g}$ of fat to survive the duration of hibernation (median $=0.48 \mathrm{~g}$, mean $=0.45 \mathrm{~g}, s d=0.12 \mathrm{~g}$, Figure $\mathrm{S} 2$ ). When considering the amount of fat bats had pre-hibernation, the median bat emerged with $1.85 \mathrm{~g}$ of body fat remaining ( mean $=2.16 \mathrm{~g}, s d=1.01 \mathrm{~g}$ ) and the heaviest $95^{\text {th }}$ percentile of bats had up to $4.05 \mathrm{~g}$ of fat remaining. Considering these residual fat values in the terms of days spent in hibernation, the median bat would have sufficient fat resources to survive an additional 181 days ( mean $=190.83$ days, $s d=45.35$ days) and those bats with $<4 \mathrm{~g}$ of fat remaining could be capable of surviving another 280 days in those optimal roosting conditions.

When $P$. destructans infection was included into the optimal roosting conditions, the median value of fat required to survive hibernation was increased by $0.7 \mathrm{~g}$ to $1.21 \mathrm{~g}$ ( mean $=1.16 \mathrm{~g}, s d=0.45)$ and the residual fat values dropped to a median of $1.22 \mathrm{~g}$ ( mean $=1.45 \mathrm{~g}, s d=0.76)$. In total, $95 \%$ of bats were predicted to emerge with between $0.61 \mathrm{~g}$ and $2.93 \mathrm{~g}$ of fat remaining after the hibernation. Translating the fat values into days, the median value was reduced $\sim 135$ days to 45.63 days (mean $=55.41$ days, $s d=45.35$ days). Mortality of bats prior to the end of the hibernation period was predicted in $4.82 \%$ of the cells where the survival of hibernating bats fell $<0$, visible in the northeastern provinces of Canada (Figure S2).

Most of the predicted best available subterranean hibernacula were expected to have available temperatures at or above $4^{\circ} \mathrm{C}$. Despite this, $32.64 \%$ of cells fell below that temperature and $6.44 \%$ of cells fell below the lower critical temperature of $2^{\circ} \mathrm{C}$ that M. lucifugusdefends during hibernation (Figure S3). Despite these lower than optimal temperatures, M. lucifugus was predicted to survive across most of its distribution, with only $0.5 \%$ of cells predicted to fall below the survival threshold - primarily around Denali National Park in Alaska. Median fat required to survive hibernation as an uninfected bat dropped $0.71 \mathrm{~g}$ to $1.11 \mathrm{~g}$ ( $m e a n=$ $1.41 \mathrm{~g}, s d=0.93)$ with a nearly identical $95 \%$ interior range. When including infection withP. destructans, the hibernation energetics model predicted the median fat required for hibernation increased $0.72 \mathrm{~g}$ to $1.21 \mathrm{~g}$, $($ mean $=1.20 \mathrm{~g}, s d=0.40 \mathrm{~g})$. Like optimal roosting conditions, infection resulted in $4.74 \%$ of cells falling below the survival threshold.

While neither of the considered hibernation conditions predict large areas to result in mortality, the increased percent of fat needed to hibernate with $P$. destructans highlights the metabolic consequences of infection (Figure S4). At 4@C and 98\% relative humidity, bats were predicted to expend a median of $154 \%$ more body fat resources to hibernate while infected for the same winter duration (Table S4). Overall, $95 \%$ of infected bats are predicted to increase their metabolic expenditure between $\sim 6 \%$ and $195 \%$ compared to their healthy counterparts. Hibernating at the predicted best available temperature suggested similar increases in the energy expended; however, the geographic distribution of where the greatest increases occurred were different than those observed under static preferred conditions.

\section{DISCUSSION}

Here we examined the spatial variation in hibernation duration and estimated pre-hibernation fat stores across North America and applied these estimates to an updated model of hibernation energetics (Haase et al. 2019) to estimate the overwinter fat necessary for M. lucifugus to survive the hibernation. By comparing the required fat and the fat available, we predicted survival of $M$. lucifugus across its distribution for two different ecological situations: one in which bats roost within their most preferred conditions and one in which they roost in the best conditions predicted to be available. Finally, we modelled the possible impact of $P$. destructans infection to understand the implications for $M$. lucifugus populations where bats have yet to be impacted by WNS. 
Winter hibernation duration is a key determinant of the overwintering survival for any hibernating species. While local variation in winter onset due to unique landscape features may create refugia where bats may persist later than or emerge from hibernation earlier than average, the lack of any previous broad-scale estimates for this critical variable highlights the need for this study. Prior work (Humphries et al. 2002, Hayman et al. 2016) had defined the hibernation period a priorias the yearly number of nights with a mean nightly temperature below freezing. Our results suggest that substantial improvements in the estimation of hibernation duration can be made by including elevation, latitude, and the number of days with frost. The number of days of frost, rather than the number of days below freezing, and the counter-intuitive negative coefficients for both Northing (-0.45) and DEM (-0.04) in the model suggest that there is more nuance to the relationship between bats and low temperatures than currently understood. Our model is potentially biased in part by the over-representation of Canadian data, low elevation sites, and the collinearity of elevation and latitude with other explanatory variables. Notably, however, when included in univariate models, the coefficient signs regress as expected with the coefficients increasing with latitude and elevation; yet the univariate models do not predict hibernation duration as well and had higher AIC scores. Because we were interested in prediction, we kept the best model by AIC; however, clearly more work is required to understand what predicts overwinter duration in bats. Also, there were few estimates of hibernation duration from the western-most US states and the more southern latitudes within the species' distribution. These factors likely impacted model results in unpredictable ways and highlights the need for additional data collection.

Species such as $M$. lucifugus may not hibernate across their broadest summer distribution, but likely seek out locations with more favourable conditions to overwinter. The maximum winter hibernation duration predicted within the distribution of $M$. lucifugus was a month and a half longer than the longest observation within our dataset, and this did not consider the portion of the species' distribution that extended into the Arctic Circle. In all probability, bats likely do not overwinter within these regions for multiple reasons. First, while our survival models predict that an uninfected bat could survive the longest predicted winter hibernation, the necessary roosting microclimate conditions may not exist on the landscape (Perry 2013, McClure et al. 2020). A series of complex interactions between surface features (e.g., slope, aspect, elevation, suitable crevices) or cave features (e.g., number of entrances, air flow, depth) ultimately define when and where suitable hibernacula conditions exist (Perry 2013, McClure et al. 2020). These site level determinants of microclimate conditions make it difficult to define a relationship between landscape-level features and the available subterranean conditions and create challenges in attempting to predict where suitable hibernacula conditions exist (McClure et al. 2020). Despite this, our use of modelled subterranean temperatures offers an improvement over assuming either static optimal conditions or mean annual surface temperature used in Hayman et al. 2016. Second, and perhaps more importantly, regions where extended winters reduce the summer active period to $<100$ days likely create significant challenges to reproductive success. With a gestation period of 60 days (O'Farrrell and Studier 1973, Kurta et al. 1989), a female bat would be hard pressed to gestate, nurse, and wean young, while still allowing the young of the year time to fatten sufficiently to survive such an extended hibernation period. These results highlight the fact that only a subset of summer distributions may be suitable for overwinter survival, an idea rarely considered in the definition of bat species' distributions.

Localized clines in body size and body mass of $M$. lucifugus have previously been recorded (Lausen et al. 2008, Lacki et al. 2015). When we compare more detailed metrics of body composition (lean mass and body fat content), however, these clines are better understood: the lean mass of bats generally stays consistent, while the difference in body mass is due to increases in fat. Our model selection suggests that variation in the duration of winter hibernation may in part drive variation in both body mass and fat stores across the range of the species. The relationships between mass (and thus fat) and latitude and days below freezing also suggest stronger selection pressure for heavier bats in more extreme conditions. While still useful, the relationship between the selected abiotic variables and body mass of bats showed strong spatial autocorrelations among residuals, and there may be additional continental-scale drivers or local determinants not investigated in this study.

The scaling relationship identified between pre-hibernation fat stores and body mass is a departure from 
the fixed 30\% value used in previous energetic modelling studies (Humphries et al. 2002, Hayman et al. 2016) and is supported by contemporary findings (Cheng et al. 2019). More localized body mass-body fat relationships may exist, yet without increased data resolution drivers of the true relationship will remain difficult to assess. Here we assumed bats did not forage during hibernation, but some bats (primarily from southern hibernacula) have been known to forage over winter (Bernard and McCracken 2017). Without data, we were required to assume bats relied exclusively on pre-hibernation fat stores.

For our modelled roost conditions, virtually all uninfected $M$. lucifugus could survive the estimated duration of winter. Observed rates of survival among uninfected overwintering bats is high (Boyles and Brack 2009) and bats are likely capable of overwintering across most of their summer distribution where suitable hibernacula exist. These results are an improvement over previous models which did not predict survival where hibernation duration $>6$ months (Hayman et al. 2016) despite our prediction that some $51 \%$ of the study extent may experience winters longer than that. In previous models, roosting microclimatic space was derived exclusively from surface metrics (i.e., mean annual surface temperature and relative humidity). Our use of a static, optimal roosting scenario served as a baseline for a best available temperature scenario and provided a more biologically relevant conditions for $M$. lucifugus, as bats are known to preferentially roost in these conditions when available (Thomas and Cloutier 1992, Haase et al. 2019). In areas where WNS has devastated hibernating colonies of $M$. lucifugus, available microclimates are often warmer with temperatures reaching $10^{\circ} \mathrm{C}$ (Perry 2013). This warmer microclimate has a significant impact on energy expenditure during hibernation (Figure S4). Despite this, our predictions for overwinter survival using estimates of the best roosting temperatures available suggest that survival may still be possible, if bats use the coldest areas within the cave or mine system. In a rare ray of hope, all of the hibernation temperatures that we recorded in the West were well below the $10^{\circ} \mathrm{C}$ mark, with hibernating M. lucifugus in northeastern Alberta and Northwest Territories roosting at about $2 @ \mathrm{C}$ and $100 \%$ relative humidity (CLL, unpublished data). While the high relative humidity is generally beneficial to the growth of $P$. destructans (and thereby a promoter of WNS pathology), the cooler temperatures may slow fungal growth in comparison to the warmer roosts of the Eastern United States.

Our model predicted uninfected bats to emerge from hibernation with remaining fat quantities far greater than the amount of fat thought to be needed to hibernate for the entire duration of winter. Some of this may be an artifact of our modelling, as we may be missing additional energetic costs such as flight during the arousal periods or sex-related differences, and we did not model deviance from the most optimal arousal patterns that can result from arousals of individuals sharing the hibernacula (neighbour-initiated arousals; Hayman et al. 2010, Jonasson and Willis 2012, Turner et al. 2015, Czenze et al. 2017). Alternatively, remaining fat stores may be retained as a buffer against adverse abiotic conditions experienced after emergence, especially among females that undergo pregnancy immediately upon exit from hibernation (Johnson et al. 2017, Czenze et al. 2017) and whose reproductive success stands to benefit from a longer growing season. Early emergence would provide a selective advantage to the young of the year, as even one or two extra weeks foraging on the landscape could increase fat stores, making them more prepared for hibernation (Reynolds and Kunz in press). Currently, little is known about the energetic demands of the emergent bats as they return to the landscape, and accurate parameterization of this factor could significantly change our definition of survival capacity and increase our estimates of WNS-related mortality.

Modelled hibernation survival predictions of bats infected with P. destructans at either $4 @ \mathrm{C}$ and $98 \%$ relative humidity or using the best available temperature do not match observations from the Eastern United States where mass mortality events have occurred due to WNS (Blehert et al. 2009, Frick et al. 2010, 2015) $(23,24,54)$. While our analysis did demonstrate infection with $P$. destructansdramatically increases the amount of energy expended during hibernation, nearly $95 \%$ of all cells analysed predicted survival despite infection. Interestingly, however, the areas with the greatest increase in hibernation energy expenditure using the best available temperature were much more in line with regions where bat populations have experienced the greatest mortality.

Irrespective of the absolute values for survival predicted within this work, our modelling predicts that $M$. 
lucifugus populations where WNS is currently absent, especially those along the Rocky Mountains, Alberta, British Columbia, and Alaska will require similar increases in energetic expenditure as populations currently impacted by WNS. Thereby, if the increase in energy expenditure results in the same pattern of mortality, M. lucifugus populations in western North America may be expected to suffer mortality events similar to those experienced in the eastern and central populations (Frick et al. 2010, 2015). The long duration winters, especially in northern British Columbia, northern Alberta, Alaska, Yukon, Northwest Territories, and portions of the Rocky Mountains, may result in severe WNS-associated pathology and disruption to hibernation physiology, although use of cool available hibernacula microclimates in some of these areas may slow fungal growth (Reeder et al. 2012, Verant et al. 2014). Identifying and describing roosting microclimates in these areas will allow for better predictive models at a regional scale. Some northern hibernacula may provide a refugia for $M$. lucifugus infected with WNS; however, this cannot be defined until the availability of microclimates and spring energy requirements are quantified. For example, if gleaning of arthropod prey in spring is typically required for successful reproduction in northern latitude $M$. lucifugus (Talerico 2008, Kaupas and Barclay 2017), then the extra energy expenditure associated with gleaning (Norberg et al. 1987) may require additional fat stores and WNS-related mortality rate may in fact be higher than predicted. Additionally, even if WNS related mortality is not directly observed, reproductive success may decline resulting in more long-term population decline.

The outputs of the energetic model that we applied are sensitive to a number of parameters and assumptions (Haase et al. 2019). The hibernation energetic model is largely derived from first principles and deviations in parameters, especially those defining the frequency or duration of arousal (cluster-based arousal, partial arousals, disturbances, etc. 8) or increasing these costs (e.g. increased number of mid-winter flights or disease pathophysiology), have the potential to alter the amount of resources required to survive hibernation. Roost microclimates are important to survival predictions and impact bat energetic and fungal growth dynamics (Marroquin et al. 2017), and further study is required to understand how relative humidity may vary across the landscape and within hibernacula.

Overall, this work represents an effort to iteratively refine both individual and landscape models of bat hibernation physiology and the impacts of WNS on bat populations. By specifically addressing the spatial heterogeneity of both abiotic phenomena and host traits, we offer some of the most detailed predictions for the potential impacts of WNS on M. lucifugus as P. destructans continues to spread through western North America.

\section{Ethics Statement}

All procedures were approved by the Texas Tech University Institutional Animal Care and Use Committee (protocol 16031-05) and permits from the Montana Department of Fish, Wildlife \& Parks (permits 2016-104, 2017-018, and 2018-008).

\section{Funding}

This project has been funded in part with Federal funds from the Department of Defence Environmental Research and Development Program (SERDP), under Contract Number W912HQ-16-C-0015 to Wildlife Conservation Society; U.S. Fish and Wildlife Service grant F17AP00593 to Wildlife Conservation Society Canada; Alberta Conservation Association grant 030-00-90-272 to Wildlife Conservation Society Canada; Royal Society Te Aparangi, grant number MAU1701 to DTSH; and funding from Texas Tech University to LPM.

\section{Acknowledgements}

For contributing additional data, we thank: Karen Blejwas (Alaska Department of Fish and Game), Tom Jung (Yukon Department of Environment), Joanna Wilson (Northwest Territories Environment and Natural Resources), Sharon Irwin (Wood Buffalo National Park), and Wildlife Conservation Society Canada's BatCaver program (analyst Jason Rae); for assistance in the field, Chuck Priestley, Cory Olson, Dave Critchley, Dave Hobson, Erin Low, Greg Horne, Heather Gates, Lisa Wilkinson, Mike Kelly, David Bobbit, Lauri 
Hanauska-Brown and many others. Any opinions, findings, and conclusions or recommendations expressed in this publication are those of the author(s) and do not necessarily reflect the views of the Government.

\section{Data availability}

All data pertaining to the work are available publicly, included as supplementary materials with this publication or available by request. All custom code for this project is available at github.com/cReedHranac/winTor and the energetic model code is available from github.com/cReedHranac/batwintor.

\section{Author Contributions}

CRH, DTSH, LPM, SHO, and CLL conceptualized the study, CGH and NWF collected field data for the project and LPM and CLL provided additional data and liaised with external research groups. CRH lead the data analysis with assistance from CGH, DTSH, and JCM. MLC created the hibernacula temperature model. All authors provided critical review and editing to the manuscript.

\section{References}

Anderson, S. C. and Ward, E. J. 2019. Black swans in space: modeling spatiotemporal processes with extremes. - Ecology 100: e02403.

Bernard, R. F. and McCracken, G. F. 2017. Winter behavior of bats and the progression of white-nose syndrome in the southeastern United States. - Ecology and Evolution 7: 1487-1496.

Bivand, R. S. and Wong, D. W. S. 2018. Comparing implementations of global and local indicators of spatial association. - TEST 27: 716-748.

Blehert, D. S. et al. 2009. Bat white-nose syndrome: An emerging fungal pathogen? - Science 323: 227-227.

Boyles, J. G. and Brack, V. 2009. Modeling survival rates of hibernating mammals with individual-based models of energy expenditure. - J Mammal 90: 9-16.

Boyles, J. G. et al. 2007. Energy availability influences microclimate selection of hibernating bats. - Journal of Experimental Biology 210: 4345-4350.

Burnham, K. and Anderson, D. R. 2002. Model Selection and Multimodel Inference - A Practical. Springer-Verlag.

Carey, C. 2019. Why do hibernators periodically arouse? - CRC Press.

Cheng, T. L. et al. 2019. Higher fat stores contribute to persistence of little brown bat populations with white-nose syndrome. - Journal of Animal Ecology 88: 591-600.

Cryan, P. M. and Wolf, B. O. 2003. Sex differences in the thermoregulation and evaporative water loss of a heterothermic bat,Lasiurus cinereus, during its spring migration. - Journal of Experimental Biology 206: 3381-3390.

Czenze, Z. J. et al. 2013. Staying cold through dinner: cold-climate bats rewarm with conspecifics but not sunset during hibernation. - J Comp Physiol B 183: 859-866.

Czenze, Z. J. et al. 2017. Thrifty females, frisky males: Winter energetics of hibernating bats from a cold climate. - Physiological and Biochemical Zoology 90: 502-511.

Fenton, M. B. and Barclay, R. M. R. 1980. Myotislucifugus . - Mammalian Species: 1-8.

French, A. R. 1985. Allometries of the durations of torpid and euthermic intervals during mammalian hibernation: A test of the theory of metabolic control of the timing of changes in body temperature. - J Comp Physiol B 156: 13-19.

Frick, S. E. and Hijmans, R. J. 2017. Worldclim 2: New 1-km spatial resolution climate surfaces for global land areas. - Internation Journal of Climatology in press. 
Frick, W. F. et al. 2010. An emerging disease causes regional population collapse of a common North American bat species. - Science (New York, N.Y.) 329: 679-82.

Frick, W. F. et al. 2015. Disease alters macroecological patterns of North American bats. - Global Ecology and Biogeography 24: 741-749.

Haase, C. G. et al. 2019. Incorporating evaporative water loss into bioenergetic models of hibernation to test for relative influence of host and pathogen traits on white-nose syndrome. - PLOS ONE 14: e0222311.

Haase, C. G. et al. 2021. Body mass and hibernation microclimate may predict bat susceptibility to whitenose syndrome. - Ecology and Evolution 11: 506-515.

Hayman, D. T. S. et al. 2010. Long-term survival of an urban fruit bat seropositive for Ebola and Lagos bat viruses. - PLoS ONE 5: 2008-2010.

Hayman, D. T. S. et al. 2016. Environment, host, and fungal traits predict continental-scale white-nose syndrome in bats. - Science Advances 2: e1500831.

Hijmans, R. J. 2016. raster : Geographic Data Analysis and Modeling. - R package version 2.5-8 in press.

Hock, R. J. 1951. The metabolic rates and body temperatures of bats. - The Biological Bulletin 101: 289-299.

Holm, S. 1979. A simple sequentially rejective multiple test procedure. - Scandinavian Journal of Statistics 6: $65-70$.

Humphries, M. M. et al. 2002. Climate-mediated energetic constraints on the distribution of hibernating mammals. - Nature 418: 313-316.

Johnson, J. S. et al. 2017. Migratory and winter activity of bats in Yellowstone National Park. - Journal of Mammalogy 98: 211-221.

Jonasson, K. A. and Willis, C. K. R. 2012. Hibernation energetics of free-ranging little brown bats. - J. Exp. Biol. 215: 2141-2149.

Kaupas, L. A. and Barclay, R. M. R. 2017. Temperature-dependent consumption of spiders by little brown bats (Myotislucifugus), but not northern long-eared bats (Myotisseptentrionalis ), in northern Canada. Canadian Journal of Zoology in press.

Klüg-Baerwald, B. J. and Brigham, R. M. 2017. Hung out to dry? Intraspecific variation in water loss in a hibernating bat. - Oecologia 183: 977-985.

Klüg-Baerwald, B. J. et al. 2017. Home is where you hang your bat: winter roost selection by prairie-living big brown bats. - J Mammal 98: 752-760.

Kunz, T. H. et al. 1998. Changes in body mass and fat reserves in pre-hibernating little brown bats (Myotis lucifugus ). - Ecoscience 5: 8-17.

Kurta, A. et al. 1989. Energetics of pregnancy and lactation in free-ranging little brown bats (Myotis lucifugus ). - Physiological Zoology 62: 804-818.

Lacki, M. J. et al. 2015. Temporal changes in body mass and body condition of cave-hibernating bats during staging and swarming. - Journal of Fish and Wildlife Management 6: 360-371.

Lane, J. E. et al. 2012. Delayed phenology and reduced fitness associated with climate change in a wild hibernator. - Nature 489: 554-557.

Langwig, K. E. et al. 2015. Host and pathogen ecology drive the seasonal dynamics of a fungal disease, white-nose syndrome. - Proceedings of the Royal Society of London B: Biological Sciences 282: 20142335. 
Langwig, K. E. et al. 2016. Drivers of variation in species impacts for a multi-host fungal disease of bats. Phil. Trans. R. Soc. B 371: 20150456.

Lausen, C. L. and Barclay, R. M. R. 2006. Winter bat activity in the Canadian prairies. - Can. J. Zool. 84: 1079-1086.

Lausen, C. et al. 2008. Beyond mtDNA: Nuclear gene flow suggests taxonomic oversplitting in the little brown bat (Myotislucifugus ). - Canadian Journal of Zoology 86: 700-713.

Lilley, T. M. et al. 2016. White-nose syndrome survivors do not exhibit frequent arousals associated with Pseudogymnoascusdestructans infection. - Front Zool in press.

Marroquin, C. M. et al. 2017. Effect of humidity on development of Pseudogymnoascus destructans, the causal agent of bat white-nose syndrome. - Northeastern Naturalist 24: 54-64.

McClure, M. L. et al. 2020. Linking surface and subterranean climate: implications for the study of hibernating bats and other cave dwellers. - Ecosphere 11: e03274.

McGuire, L. P. and Guglielmo, C. G. 2010. Quantitative magnetic resonance: a rapid, non-invasive body composition analysis technique for live and salvaged bats. - J Mammal 91: 1375-1380.

McGuire, L. P. et al. 2014. Bats on a budget: torpor-assisted migration saves time and energy. - PLoS One in press.

McGuire, L. P. et al. 2018. Common condition indices are no more effective than body mass for estimating fat stores in insectivorous bats. - Journal of Mammalogy 99: 1065-1071.

Mellanby, K. 1939. Low temperature and insect activity. - Proceedings of the Royal Society B: Biological Sciences 127: 473-487.

Norberg, U. M. et al. 1987. Ecological morphology and flight in bats (Mammalia; Chiroptera): wing adaptations, flight performance, foraging strategy and echolocation. - Philosophical Transactions of the Royal Society of London. B, Biological Sciences 316: 335-427.

Norquay, K. J. O. and Willis, C. K. R. Hibernation phenology of Myotis lucifugus. - Journal of Zoology 294: 85-92.

O'Farrrell, M. J. and Studier, E. H. 1973. Reproduction, growth, and development in Myotis thysanodes and M. lucifugus(Chiroptera: Vespertilionidae). - Ecology 54: 18-30.

Pebesma, E. and Bivand, R. 2005. Classes and methods for spatial data in R. - R News 5: 9-13.

Perry, R. W. 2013. A review of factors affecting cave climates for hibernating bats in temperate North America. - Environmental Reviews 21: 28-39 21: 28-39.

R Development Core Team 2009. R: a language and environment for statistical computing R Foundation for Statistical Computing.

Reeder, D. M. et al. 2012. Frequent arousal from hibernation linked to severity of infection and mortality in bats with white-nose syndrome. - PLOS ONE 7: e38920.

Reynolds, S. and Kunz, T. H. Changes in body composition during reproduction and postnatal growth in the little brown bat, Myotislucifugus (Chiroptera: Vespertilionidae). - Ecoscience 7: 10-17.

Ruf, T. and Geiser, F. 2015. Daily torpor and hibernation in birds and mammals. - Biological Reviews 90: 891-926.

Speakman, J. R. and Rowland, A. 1999. Preparing for inactivity: how insectivorous bats deposit a fat store for hibernation. - Proc Nutr Soc 58: 123-131. 
Speakman, J. R. and Thomas, D. W. 2003. Physiological ecology and energetics of bats. - In: Kunz, T. H. and Fenton, M. B. (eds), Bat Ecology. First. University Of Chicago Press, pp. 430-490.

Talerico, J. M. 2008. The behaviour, diet and morphology of the little brown bat (Myotis lucifug us) near the northern extent of its range in Yukon Canada.

Thomas, D. W. and Cloutier, D. 1992. Evaporative water loss by hibernating little brown bats, Myotis lucifugus . - Physiological Zoology 65: 443-456.

Thomas, D. W. et al. 1990. Winter energy budgets and cost of arousals for hibernating little brown bats, Myotis lucifugus. - Journal of Mammalogy 71: 475-479.

Turner, J. M. et al. 2015. Conspecific disturbance contributes to altered hibernation patterns in bats with white-nose syndrome. - Physiol. Behav. 140: 71-78.

Verant, M. L. et al. 2012. Temperature-dependent growth ofGeomyces destructans, the fungus that causes bat white-nose syndrome. - PLOS ONE 7: e46280.

Verant, M. L. et al. 2014. White-nose syndrome initiates a cascade of physiologic disturbances in the hibernating bat host. - BMC Physiology 14: 10.

Wang, L. C. H. 1978. Energetics and field aspects of mammalian torpor: the Richardson's ground squirrel. In: Wang, L. C. H. and Hudson, J. W. (eds), Strategies in the Cold. Academic Press, pp. 109-145.

Wang, T. et al. 2016. Locally downscaled and spatially customizable climate data for historical and future periods for North America. - PLOS ONE 11: e0156720.

Warnecke, L. et al. 2012. Inoculation of bats with EuropeanGeomyces destructans supports the novel pathogen hypothesis for the origin of white-nose syndrome. - Proceedings of the National Academy of Sciences 109: 6999-7003.

Willis, C. K. R. et al. 2006. Deep, prolonged torpor by pregnant, free-ranging bats. - Naturwissenschaften 93: $80-83$.
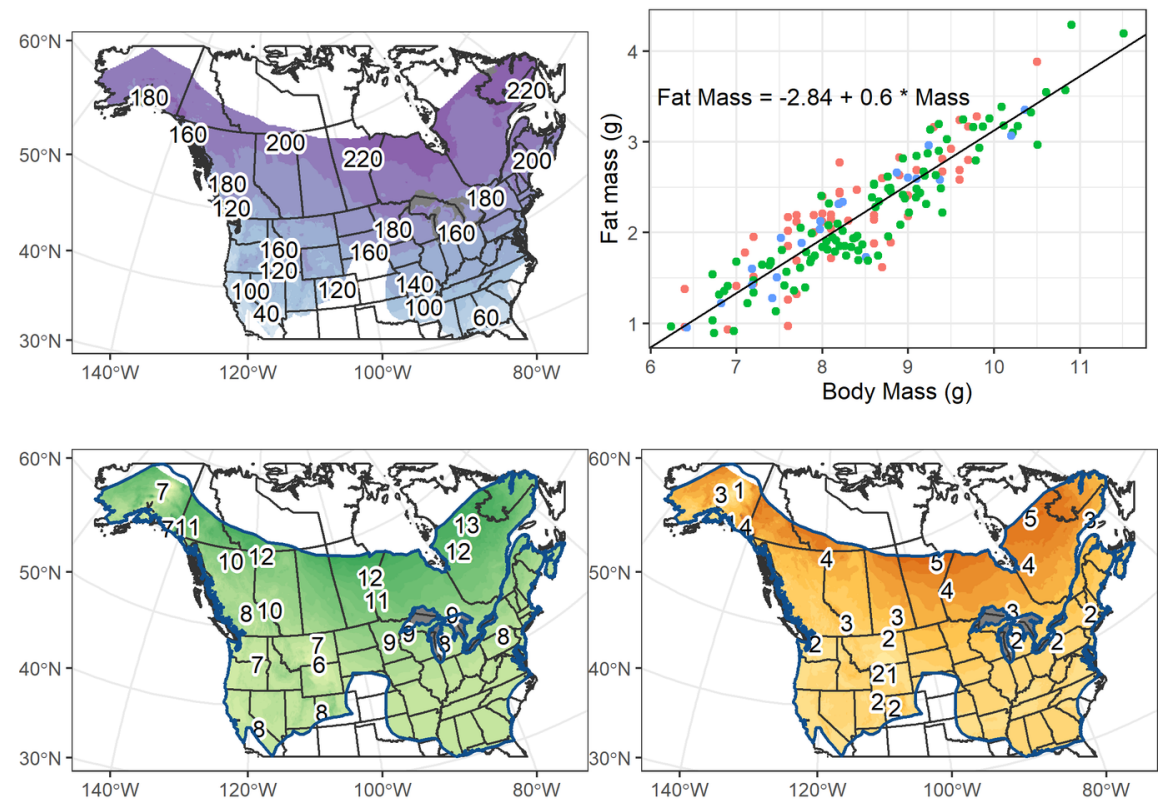

Figure 1. Predicted hibernation duration, fat and body mass forMyotis lucifugus. Top left: 
Predicted hibernation duration (days) across temperate North America for Myotis lucifugus . Top right: Linear relationship between body fat and body mass. Each point represents an individual bat and colours indicate the state of each record (Montana $=$ red, New York $=$ green, Vermont $=$ blue). Bottom left: Predicted pre-hibernation body mass of $M$. lucifugus (g) across the species' distribution. Bottom right: Predicted pre-hibernation fat resources $(\mathrm{g})$ from the linear relationship between fat mass and body mass. Numbers define changes in contours.
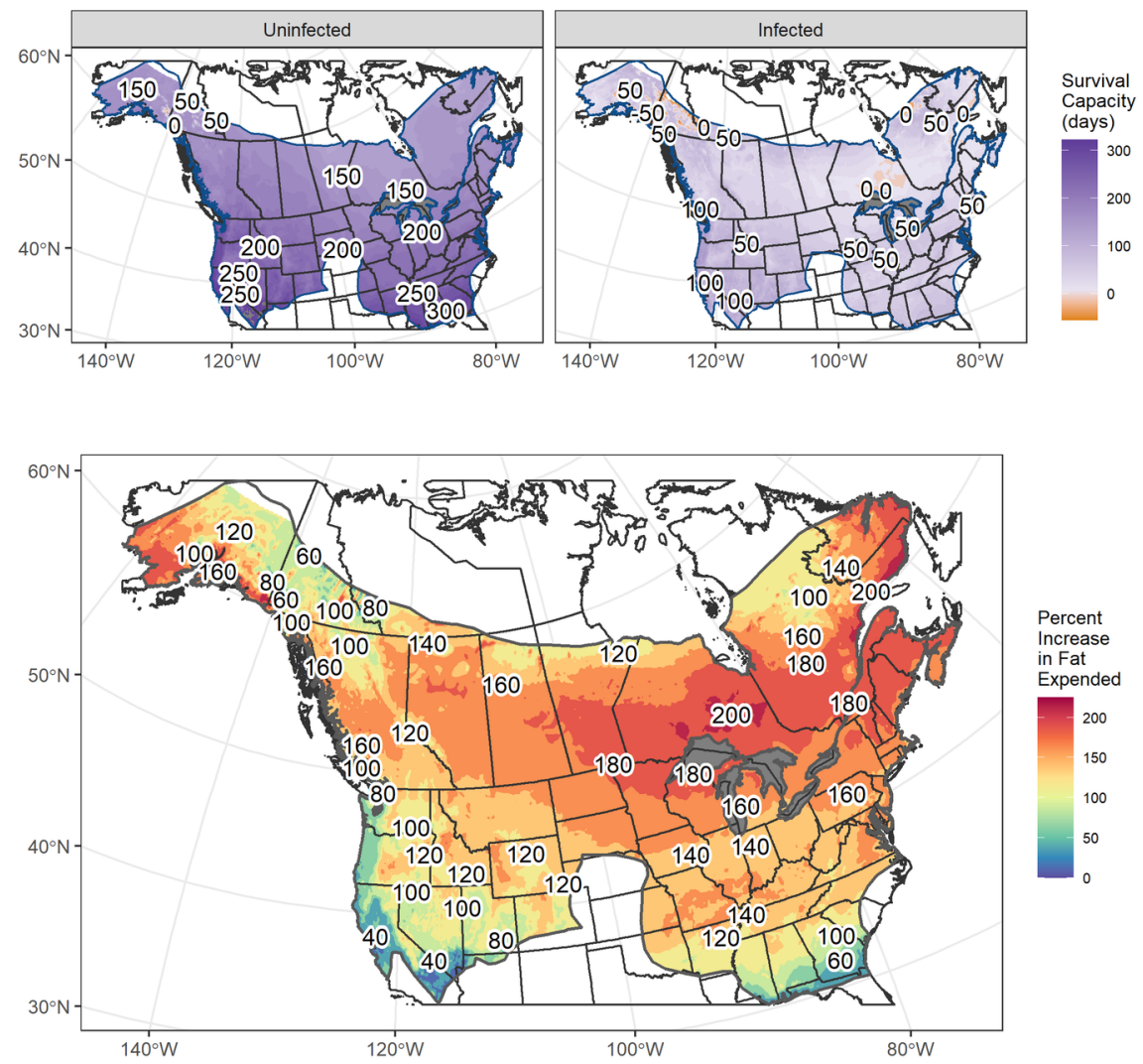

Figure 2. Top row: Predicted survival (days) for healthy (top left) and infected (top right) Myotis lucifugushibernating at the best available temperatures $\left({ }^{\circ} \mathrm{C}\right)$ in caves or mines and $98 \%$ relative humidity. Survival was calculated by subtracting the maximum days in hibernation that an individual can tolerate based upon pre-hibernation fat stores from the predicted duration of hibernation. Positive values (purple) suggest bats will likely survive the duration of hibernation under those conditions while values below 0 imply that the fat stores were insufficient for survival. Bottom: Relative increase (\%) in fat stores used over predicted hibernation duration when infected with Pseudogymnoascus destructans compared with healthy bats when hibernating at the best available temperature predicted to occur within caves and mines at $98 \%$ relative humidity. 